\title{
Did the number of older adults with frailty increase during the COVID-19 pandemic? A prospective cohort study in Japan
}

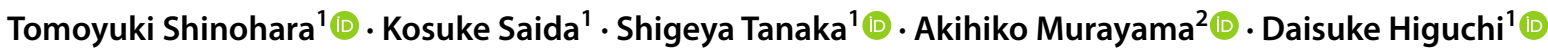

Received: 11 March 2021 / Accepted: 19 May 2021 / Published online: 3 June 2021

(c) European Geriatric Medicine Society 2021

\section{Key summary points}

Aim This study aimed to clarify the actual frail status over 6 months with the COVID-19 countermeasures based on a prospective cohort study.

Findings Pre-frailty and frailty prevalences among Japanese community-dwelling, older adults were 55.0 and $7.9 \%$ at the baseline, and 57.3 and $11.8 \%$ at the follow-up respectively.

Frailty over the 6 months could be predicted by increasing age and multimorbidity during the COVID-19 pandemic.

Message We should prevent frailty affected by the implementation of COVID-19 countermeasures.

\begin{abstract}
Purpose To clarify the actual frail status over 6 months with the COVID-19 countermeasures.

Methods This prospective cohort study was conducted between the baseline assessment from May 11 to July 10 in 2020 , and the follow-up assessment from November 11, 2020, to January 10, 2021, in Japan. The survey forms were distributed among 1953 community-dwelling older adults. Frailty status was assessed using the Frailty Screening Index.

Results In total, 702 older adults (35.2\%) returned the survey forms, and 593 (mean age $=78.8$ years, and $77.4 \%$ females) older adults without missing values for the survey forms were analyzed. Pre-frail and frail prevalence were 55.0 and $7.9 \%$ at the baseline, and 57.3 and $11.8 \%$ at the follow-up, respectively. Frailty transition that indicated transition from robust or pre-frail at the baseline to frail at the follow-up was $9.9 \%$.

Conclusion Increase in frailty might indicate frailty related to implementation of COVID-19 countermeasures.
\end{abstract}

Keywords Frailty · COVID-19 · Older adult

\section{Introduction}

Japan needs to facilitate healthy aging to maintain functional capacity. Exercise and communication gatherings as well as regular visits to prevent anxiety and isolation among older adults were the activities recommended locally [1], as these lower the risk of the occurrence of frailty [2]. However, the

Tomoyuki Shinohara

shinohara-t@takasaki-u.ac.jp

1 Department of Physical Therapy, Faculty of Health Care, Takasaki University of Health and Welfare, 501 Naka Orui-machi, Takasaki-shi, Gunma 370-0033, Japan

2 Department of Physical Therapy, Faculty of Rehabilitation, Gunma University of Health and Welfare, K'BIX Genki21 Maebashi 6-7F, 2-12-1 Hon-machi, Maebashi-shi, Gunma 371-0023, Japan
Japanese government requested that the population avoid mass gatherings and thoroughly implement social distancing during the COVID-19 pandemic [3]. As a result, local activities for community-dwelling older adults were canceled in each community and have not been restarted yet. The changes in lifestyle and the cancelation of local activities due to COVID-19 countermeasures might thereby affect frailty in older adults. It is suggested that frailty caused by the implementation of COVID-19 countermeasures will be known as "Corona-Frailty" [4].

Previous studies reported frailty transition rates based on their longitudinal research period [5-8]. However, the frailty transition rate might be different from the norm period since no one has ever experienced the impact of COVID-19 countermeasures earlier and thus, it cannot be predicted. In fact, the COVID-19 countermeasures, such as self-restraint (excursions, eating out, shopping, and travel), 
implementation of telework by companies, and restrictions on visiting hospitalized individuals, were enforced on people during the time points for the baseline and follow-up assessments for this study. The number of positive COVID-19 cases in June 2020 and December 2020 were 1748 (0.001\% of the total population in Japan) and 85,891 (0.068\%), respectively [9]. The lockdown was not legally enforceable; therefore, the Japanese government and administration requested self-restraint. Although there is a lot of research on patients with COVID-19, there is a lack of research on the impact of COVID-19 on frailty in community-dwelling older adults.

It is important to understand the actual frailty and factors associated with frailty transition to take measures for frailty in the future. Therefore, this brief report aimed to clarify the actual frailty status over 6 months with COVID-19 countermeasures and in association with the frailty status transition.

\section{Methods}

\section{Study design and participants}

A prospective cohort study was conducted in Takasaki City, Gunma Prefecture, Japan with 1953 community-dwelling older adults aged 65 and over, living in local housing and who were helped regularly by local volunteers being eligible for inclusion. Whether the participants had been infected with COVID-19 or were following special preventive confinement measures was not verified. As per the exclusion criteria, care home residents' patients with stroke or cancer were not included as participants. The survey forms were distributed to the target population by local volunteers or support professionals who helped them regularly. If they agreed to participate in the study, they were required to write their name on the survey form and return it via post. The survey form included questions concerning age, gender, morbidity, living arrangement (with cohabitant or alone), and frailty status. Multimorbidity was defined as having more than one chronic disease [10]. The baseline assessment was carried out from May 11 to July 10, 2020, and the follow-up assessment from November 11, 2020, to January 10, 2021.

This study was approved by the Research Ethics Committee of Takasaki University of Health and Welfare (approval number: 2009) and was registered with the University Hospital Medical Information Network (UMIN000040335).

\section{Measurements}

Frailty was assessed using the Frailty Screening Index (FSI) [11] that had predictive validity for disability [11] and concurrent validity for social frailty [12] among Japanese older adults. The FSI is a questionnaire that comprises five items;
"Have you lost $2 \mathrm{~kg}$ or more in the past six months?", "Do you think you walk slower than before?", "Do you go for a walk for your health at least once a week?", "Can you recall what happened five minutes ago?", and "In the past two weeks, have you felt tired without reason?". These items are answered with a simple Yes/No response, and one point is scored if an answer indicates a frail condition. The total score of the FSI ranges from 0 to 5 . The frailty status was based on the participant's score, wherein a score of 3 or more was defined as frail; $1-2$ as pre-frail, and 0 as robust [11].

\section{Statistical analysis}

Descriptive statistics for the FSI score were presented and compared between the baseline and follow-up using the Wilcoxon signed-rank test. Ordinal logistic regression analysis was then performed for univariate analysis to identify sociodemographic factors associated with three frailty status at the follow-up; robust, pre-frail, or frail, and for multivariate analysis with adjusted frailty status at the baseline. Statistical analyses were performed using IBM SPSS Statistics for Windows, version 26 (IBM Corp., Armonk, N.Y., USA), or R version 4.0.0, with $p<0.05$ indicating statistical significance.

\section{Results}

Out of the population to which the survey form was distributed, 702 older adults returned the survey forms with written consent at both baseline and follow-up, and the total response rate was $35.9 \%$. In total, 593 older adults answered all questions of the survey form and met the inclusion criteria (Fig. 1). The mean interval between the baseline and the follow-up was $186.6 \pm 17.3$ days. Table 1 shows the participants' sociodemographic characteristics. At the baseline, $37.1 \%(n=220)$ participants were robust, $55.0 \%(n=326)$ were pre-frail, and $7.9 \%(n=47)$ were frail. At the follow-up after 6 months, 30.9\% $(n=183)$ of participants were robust, $57.3 \%(n=340)$ were pre-frail, and $11.8 \%(n=70)$ were frail. Furthermore, among the $92.1 \%(n=546)$ participants who were robust or pre-frail at the baseline, $9.9 \%(n=54)$ transitioned to frail at the follow-up.

Figure 2 shows the distribution of the FSI score. At the baseline/follow-up, 220/183, 188/226, 138/114, 38/50, 8/19, and $1 / 1$ participants were found to have an FSI score of 0,1 , $2,3,4$, and 5 , respectively. The modes were 0.0 and 1.0 for the baseline and follow-up, respectively. A significant difference was observed in the FSI score between the baseline and follow-up $(\mathrm{Z}=2.972, p=0.003)$.

Table 2 shows the factors associated with frailty status over 6 moths using ordinal logistic regression. The adjusted 
Fig. 1 The flowchart showing the study participants for analyses

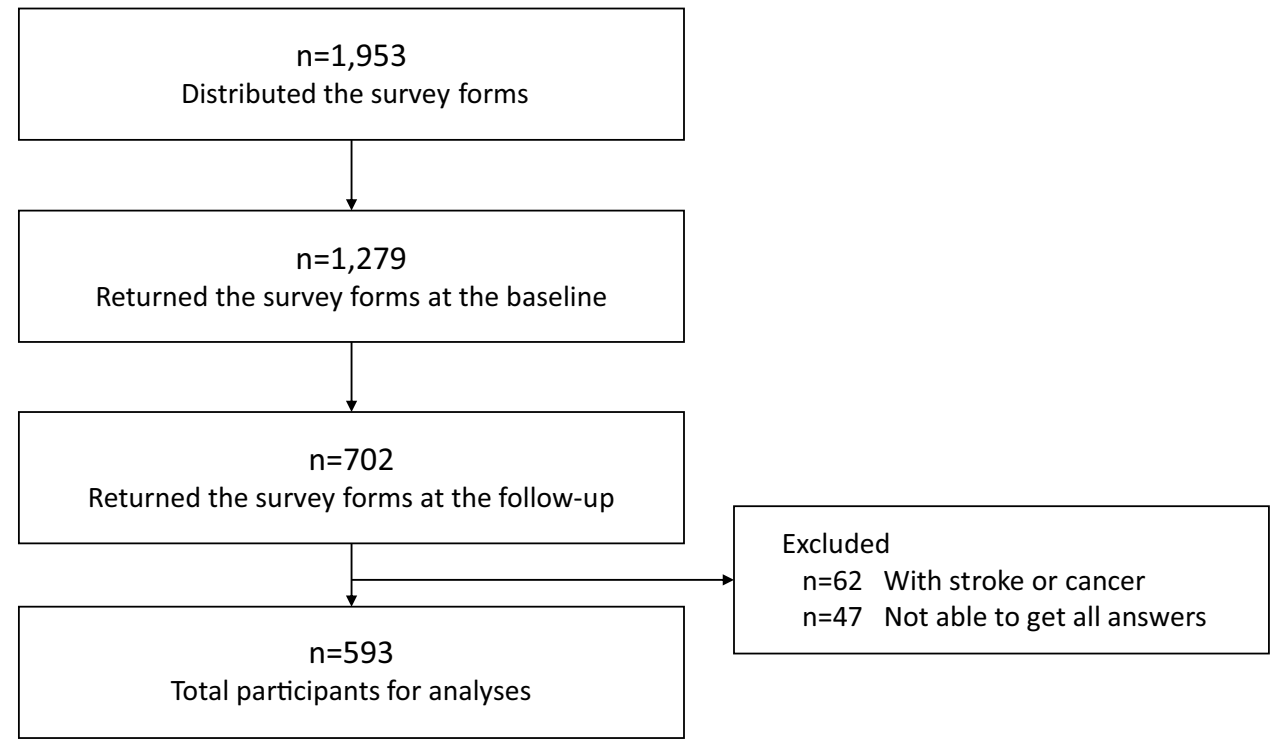

Table 1 Sociodemographic characteristics of participants

\begin{tabular}{lll}
\hline Characteristics & $n$ & $(\%)$ \\
\hline $\begin{array}{l}\text { Age, mean } \pm \text { SD (years) } \\
\text { Gender }\end{array}$ & $78.8 \pm 6.2$ & \\
Female & 459 & $(77.4)$ \\
Male & 134 & $(22.6)$ \\
Morbidity & & \\
Hypertension & 250 & $(42.2)$ \\
Osteoporosis & 95 & $(16.0)$ \\
Dyslipidemia & 83 & $(14.0)$ \\
Heart disease & 76 & $(12.8)$ \\
Diabetes mellitus & 74 & $(12.5)$ \\
Osteoarthritis & 66 & $(11.1)$ \\
Multimorbidity & 189 & $(31.9)$ \\
Living arrangement & & \\
With cohabitant & 189 & $(31.9)$ \\
Alone & 404 & $(68.1)$ \\
\hline
\end{tabular}

multivariate model revealed that frailty status at the followup was associated with increasing age (odds ratio (OR) 1.082 , 95\% confidence interval (CI) 1.050-1.115), multimorbidity (OR 1.619, 95\% CI 1.113-2.358).

\section{Discussion}

The pre-frailty and frailty prevalence were compared with a meta-analysis in Japan, and were 48.1 and $7.5 \%$, respectively [13]. Although the pre-frailty and frailty prevalence in the present study were slightly high, there was no clear difference in the tendency between the frailty prevalence for participants at baseline and in the meta-analysis. The

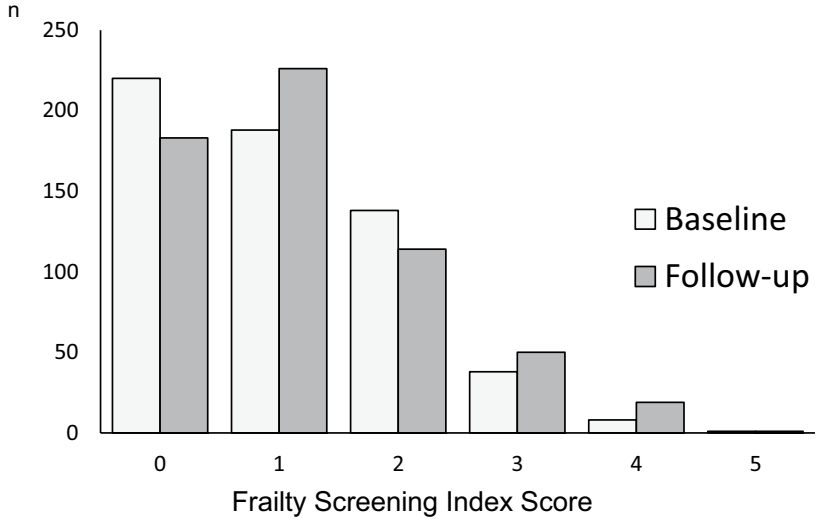

Fig. 2 The frailty screening index score are displayed between the baseline and follow-up over six months. The mode was 0.0 and 1.0 for the baseline and follow-up, respectively. There was a significant difference in the FSI score between the baseline and follow-up $(Z=2.972, p=0.003)$

participants in the present study were not considered to be uniquely community-dwelling older adults.

In the study over 2 years of follow-up, it was reported that $6.9 \%$ older adults were aged 60 years and above [8]. In another study in Japan, it was reported that $7.2 \%$ older adults' mean age was 73.0 [7]. Although the region, age, and follow-up period of the target population differed between the previous studies and the current study, the transition rate of $9.9 \%$ that was observed over 6 months in this study might be higher than previous studies conducted in the norm period. During the two time-points for baseline and follow-up assessments, the COVID-19 countermeasures were enforced on people in Japan. Among the communitydwelling older adults, physical activity decreased during the lockdown in Spain [14]. In Japan, where self-restraint was 
Table 2 Factors associated with frailty status over 6 months using ordinal logistic regression analysis

\begin{tabular}{|c|c|c|c|c|c|c|}
\hline \multirow[t]{3}{*}{ Sociodemographic factors } & \multicolumn{6}{|c|}{ Frailty status at the follow-up over 6 months } \\
\hline & \multicolumn{3}{|c|}{ Unadjusted model $^{\mathrm{a}}$} & \multicolumn{3}{|c|}{ Adjusted model $^{b}$} \\
\hline & OR & $95 \% \mathrm{CI}$ & $p$ value & OR & $95 \% \mathrm{CI}$ & $p$ value \\
\hline Age & 1.103 & $1.074-1.133$ & .000 & 1.082 & $1.050-1.115$ & .000 \\
\hline \multicolumn{7}{|l|}{ Gender } \\
\hline Female & 1.000 & (Reference) & & 1.000 & (Reference) & \\
\hline Male & 0.787 & $0.541-1.148$ & .214 & 1.051 & $0.695-1.592$ & .812 \\
\hline \multicolumn{7}{|l|}{ Multimorbidity } \\
\hline Without multimobidity & 1.000 & (Reference) & & 1.000 & (Reference) & \\
\hline Multimobidity & 2.125 & $1.498-3.016$ & .000 & 1.619 & $1.113-2.358$ & .012 \\
\hline \multicolumn{7}{|l|}{ Living arrangement } \\
\hline With cohabitant & 1.000 & (Reference) & & 1.000 & (Reference) & \\
\hline Alone & 1.231 & $0.869-1.742$ & .241 & 0.897 & $0.608-1.322$ & .582 \\
\hline
\end{tabular}

${ }^{a}$ Model for univariate analysis

${ }^{\mathrm{b}}$ Model for multivariate analysis and adjustment for frailty status at the baseline

$O R$ odds ratio; $C I$ confidence interval enforced instead of a lockdown, physical activity decreased compared to the period before the COVID-19 pandemic [15]. Decreased physical activity caused by the COVID-19 countermeasures might have increased frailty. An increase in frailty suggested in this study might indicate Corona-Frailty [4], which conveys frailty caused by the implementation of COVID-19 countermeasures.

This study had several limitations. First, the present study was not a complete enumeration or a random sampling survey. The community-dwelling older adults to whom the local volunteers or professionals could visit and distribute the survey form were included as participants. The frailty status of the present study was not considered to be unique compared to the Japanese meta-analytic study. Furthermore, sampling bias may be present. Second, we could not analyze the longitudinal frailty status compared to the homogeneous group in the norm period. Third, we assessed the frailty status using the FSI that did not require face-to-face measurement with regard to complying with social distancing as a COVID-19 countermeasure. The validity of the FSI as indicated by the relationship with other assessments involving actual measurements and sensitivity for frailty status change of the FSI should be examined in future studies.

In conclusion, during this period, the frailty transition rate indicates the transition from robust or pre-frail to frail was $9.9 \%$. An increase in frailty might indicate frailty caused by the implementation of COVID-19 countermeasures. Frailty at the follow-up after 6 months could be predicted by increasing age and multimorbidity during the COVID-19 pandemic.

Acknowledgements We would like to express our sincere gratitude to Kenichi Sudo, Munehisa Sudo, Atsushi Kuwabara, Satoshi Tanaka,
Chieko Mesaki, Junko Ishii, Kumi Aoki, Norie Torizuka, Miyuki Ogawa, Yumi Ino, Seiichi Asanuma, Izumi Tsutsumi, Susumu Shimomura, Nobuko Kaseda, Kazuaki Kuwabara, Yuriko Yoshiara, Ryo Koike, Masaaki Arai, Ayako Yamazaki, and all district welfare commissioners who cooperated. The parts of this work are supported by the Nippon Life Insurance Foundation (Grant 2020-0203-04) and the Japanese Society for the Promotion of Science KAKENHI (Grant 19K19712). We would like to thank Editage (www.editage.com) for English editing for supporting COVID-19 research.

Author contribution Study concept and design: TS. Acquisition of data: TS and KS. Analysis and interpretation of data: TS, KS, and DH. Drafting of the manuscript: TS. Critical revision of the manuscript for important intellectual content: ST, AM, and DH.

Funding The parts of this work are supported by the Nippon Life Insurance Foundation (Grant 2020-0203-04) and the Japanese Society for the Promotion of Science KAKENHI (Grant 19K19712).

\section{Declarations}

Conflict of interest The authors declare that they have no conflict of interest.

Ethics approval This study was approved by the Research Ethics Committee of the Takasaki University of Health and Welfare (Approval No. 2009).

Informed consent We obtained written informed consent from all participants prior to their participation in this study.

\section{References}

1. Tsutsui $T$ (2014) Implementation process and challenges for the community-based integrated care system in Japan. Int J Integr Care 14:e002. https://doi.org/10.5334/ijic.988 
2. Okura M, Ogita M, Yamamoto M, Nakai T, Numata T, Arai $H$ (2018) Community activities predict disability and mortality in community-dwelling older adults. Geriatr Gerontol Int 18:11141124. https://doi.org/10.1111/ggi.13315

3. Looi MK (2020) Covid-19: Japan prepares to extend state of emergency nationwide as "untraceable" cases soar. BMJ 369:m1543. https://doi.org/10.1136/bmj.m1543

4. Shinohara T, Saida K, Tanaka S, Murayama A (2020) Rapid response: impact of the COVID-19 pandemic on frailty in the elderly citizen; corona-frailty. BMJ 369:m1543. https://doi.org/ 10.1136/bmj.m1543

5. Rabassa M, Zamora-Ros R, Urpi-Sarda M, Bandinelli S, Ferrucci L, Andres-Lacueva C et al (2015) Association of habitual dietary resveratrol exposure with the development of frailty in older age: the Invecchiare in Chianti study. Am J Clin Nutr 102:1534-1542. https://doi.org/10.3945/ajcn.115.118976

6. Ramsay SE, Papachristou E, Watt RG, Tsakos G, Lennon LT, Papacosta AO et al (2018) Influence of poor oral health on physical frailty: a population-based cohort study of older British men. J Am Geriatr Soc 66:473-479. https://doi.org/10.1111/jgs.1517

7. Tanaka T, Takahashi K, Hirano H, Kikutani T, Watanabe Y, Ohara $\mathrm{Y}$ et al (2018) Oral frailty as a risk factor for physical frailty and mortality in community-dwelling elderly. J Gerontol A Biol Sci Med Sci 73:1661-1667. https://doi.org/10.1093/gerona/glx225

8. Ye B, Chen H, Huang L, Ruan Y, Qi S, Guo Y et al (2020) Changes in frailty among community-dwelling Chinese older adults and its predictors: evidence from a two-year longitudinal study. BMC geriatr 20:130. https://doi.org/10.1186/s12877-020-01530-x

9. Ministry of Health, Labour and Welfare. https://www.mhlw.go. jp/stf/covid-19/open-data.html. Accessed 30 Apr 2021

10. World Health Organization. World report on ageing and health 2015. https://www.who.int/ageing/events/world-report-2015launch/en/. Accessed 11 Mar 2021
11. Yamada M, Arai H (2015) Predictive value of frailty scores for healthy life expectancy in community-dwelling older Japanese adults. J Am Med Dir Assoc 16(1002):e7-e11. https://doi.org/10. 1016/j.jamda.2015.08.001

12. Yamada M, Arai H (2018) Social frailty predicts incident disability and mortality among community-dwelling Japanese older adults. J Am Med Dir Assoc 19:1099-1103. https://doi.org/10. 1016/j.jamda.2018.09.013

13. Kojima G, Iliffe S, Taniguchi Y, Shimada H, Rakugi H, Walters K (2017) Prevalence of frailty in Japan: a systematic review and meta-analysis. J Epidemiol 27:347-353. https://doi.org/10.1016/j. je.2016.09.008

14. Pérez LM, Castellano-Tejedor C, Cesari M, Soto-Bagaria L, Ars J, Zambom-Ferraresi F et al (2021) Depressive symptoms, fatigue and social relationships influenced physical activity in frail older community-dwellers during the spanish lockdown due to the COVID-19 pandemic. Int J Environ Res Public Health 18:808. https://doi.org/10.3390/ijerph18020808

15. Yamada M, Kimura Y, Ishiyama D, Otobe Y, Suzuki M, Koyama $S$ et al (2020) Effect of the COVID-19 epidemic on physical activity in community-dwelling older adults in Japan: a cross-sectional online survey. J Nutr Health Aging 24:948-950. https://doi.org/ 10.1007/s12603-020-1424-2

Publisher's Note Springer Nature remains neutral with regard to jurisdictional claims in published maps and institutional affiliations. 